\title{
Quantifying Maternal Blood Loss from the Hysterotomy at Cesarean Delivery
}

\author{
Rebecca M. Reimers', Daniela Carusi'1, Michaela K. Farber ${ }^{2}$, James A. Greenberg3 \\ ${ }^{1}$ Division of Maternal Fetal Medicine, Brigham \& Women's Hospital, Boston, MA, USA \\ ${ }^{2}$ Department of Anesthesiology, Perioperative and Pain Medicine, Brigham \& Women's Hospital, Boston, MA, USA \\ ${ }^{3}$ Department of Obstetrics and Gynecology, Brigham \& Women's Hospital, Boston, MA, USA \\ Email: rreimers@partners.org
}

How to cite this paper: Reimers, R.M., Carusi, D., Farber, M.K. and Greenberg, J.A. (2020) Quantifying Maternal Blood Loss from the Hysterotomy at Cesarean Delivery. Open Journal of Obstetrics and Gynecology, 10, 969-975.

https://doi.org/10.4236/ojog.2020.1080091

Received: April 30, 2020

Accepted: July 31, 2020

Published: August 3, 2020

Copyright (c) 2020 by author(s) and Scientific Research Publishing Inc. This work is licensed under the Creative Commons Attribution International License (CC BY 4.0).

http://creativecommons.org/licenses/by/4.0/

\begin{abstract}
Background: With newer protocols, such as delayed cord clamping, becoming routine practice, determining the potential maternal consequences is important. In particular, establishing normative values for blood loss from the hysterotomy would be helpful in addressing techniques to minimize total blood loss for cesarean deliveries. Objective: Blood loss from the hysterotomy during cesarean delivery has not been reported using quantitative methods. We aimed to quantify the rate of blood loss during cesarean delivery from the hysterotomy between creation and closure. Methods: This single center, prospective, case series was collected in 2018. Women with singleton pregnancies undergoing cesarean delivery at $\geq 37$ 0/7 weeks at Brigham and Women's Hospital were included. Delayed cord clamping was performed which allowed for quantification of blood loss through gravimetric methods and descriptive statistics were performed. Results: Twenty patients were included. The mean hysterotomy closure delay for cord blood collection was 47 seconds (SD 10.2) and the mean maternal blood volume collected was 110.8 $\mathrm{mL}$ (SD $53.4 \mathrm{~mL}$ ). Blood loss per minute was calculated with a median of 150 $\mathrm{mL} /$ minute (IQR $88.8 \mathrm{~mL}, 95 \%$ CI 109.2 - $190.4 \mathrm{~mL}$ ). The mean post-operative hematocrit drop was $4.4 \%$, and there were no blood transfusions. There was a single hysterotomy extension and a quantified blood loss of $413 \mathrm{~mL}$ per minute. Conclusion: We found a mean blood loss of $150 \mathrm{~mL} / \mathrm{min}$ without hysterotomy extension. With a hysterotomy extension, the blood lost per minute was more profound. This normative data can be helpful for surgical planning with regards to delayed cord clamping or cord blood collection for banking.
\end{abstract}

\section{Keywords}

Cesarean Section, Gravimetry, Blood Loss, Hysterotomy, Surgery, Humans, 
Obstetrics, Delivery

\section{Introduction}

All aspects of surgical bleeding during cesarean delivery are relevant points of intervention in order to minimize maternal morbidity from hemorrhage. While uterine atony and abnormal placentation are frequently mentioned in this discussion, normative data regarding blood loss from the non-pathologic components of this surgical procedure are lacking. With newer protocol, such as delayed cord clamping, becoming routine practice, determining the potential maternal consequences is important [1]. In particular, establishing normative values for blood loss from the hysterotomy would be helpful in addressing techniques to minimize total blood loss for cesarean deliveries. When the hysterotomy is created during a cesarean delivery, there is inevitably bleeding from the disrupted myometrial edges of the incision. Hysterotomy bleeding is highly variable depending upon whether there are venous sinuses involved or extensions into the uterine vasculature. In the late 1980's and early 1990's, there were several studies evaluating the potential hemostasis benefits of using surgical staplers on the hysterotomy; however, those trials employed only estimated rather than measured blood loss and only one reported the blood loss specifically from the hysterotomy rather than the entire procedure [2] [3] [4] [5]. In that study of 101 patients a mean blood loss of $294 \mathrm{~mL}$ was reported based on surgeons' estimates [2]. The purpose of this pilot study is to more accurately quantify the rate of blood loss that a mother incurs from the hysterotomy between creation and closure.

\section{Materials and Methods}

This prospective pilot study was approved by the Partners Human Research Committee Institutional Review Board including a waiver of informed consent given there was no significant deviation from standard care. Given the absence of prior data, a sample size of 20 patients approved as a pilot study to acquire baseline data that could be used to appropriately power future research. After approval from the delivering obstetrician, a convenience sample of women scheduled for elective or non-elective cesarean delivery at Brigham \& Women's Hospital, a single tertiary medical center between January $1^{\text {st }}$ and April $30^{\text {th }}, 2018$ were approached. Eligible women were 18 years or older with a singleton pregnancy at $370 / 7$ weeks gestational age or greater with delayed cord clamping planned at the time of delivery. A protocol for delayed umbilical cord clamping for 60 seconds during cesarean delivery has been in place at our institution since 2017. Patients ineligible for cord clamping include suspected abruption, placenta previa, vasa previa, monochorionic twins, or pediatrician request for expedited clamping to facilitate neonatal resuscitation. Women with placenta previa, sus- 
pected placenta accreta, known coagulopathy, or urgent cesarean delivery were excluded from this study.

After delivery, the surgical field was cleared of existing fluids and dry laparotomy sponges were applied to the hysterotomy. This allowed for collection of blood lost from the hysterotomy during the time of delayed cord clamping. Oxytocin is routinely administered after delivery of the infant. Total time of hysterotomy blood collection was from the placement of the first dry laparotomy sponge on the hysterotomy until the umbilical cord was clamped. Blood loss was quantitated using the Triton L\&D gravimetric system (Gauss Surgical, Menlo Park, CA). Blood-soaked sponges were weighed on an electronic scale, and the sponge dry weight subtracted for gravimetric calculation of blood loss. Intraoperative collected data included the duration of laparotomy pad application to the uterus, time of delayed cord clamping, and quantitative blood loss. Study coinvestigators directly observed the entire collection period to ensure adherence to the study protocol and collected data.

Recorded data included the date of procedure, patient demographics, indication for cesarean delivery, primary versus repeat cesarean delivery, pre-operative and postpartum day one hematocrit and platelet counts, hysterotomy blood collection time, milliliters of blood loss by gravimetry, surgeon estimated blood loss for the case, presence or absence of hysterotomy extensions, and blood transfusion.

Statistical analysis included descriptive statistics with a students' t-test for continuous variables and $\mathrm{p}$-values of $<0.05$ considered significant. Mean values are presented with standard deviation and median values are presented with interquartile range. Analyses were conducted using the statistical package SPSS version 24.0 (SPSS Inc., Chicago, IL).

\section{Results}

Twenty patients were recruited. Table 1 details the baseline patient characteristics. The average age was 32 years old, $65 \%$ of cases were repeat cesarean deliveries, and $40 \%$ of the cases were in labor at the time of cesarean delivery. The average gestational age was 38.7 weeks $( \pm 1.3)$. Pre-operative hematocrits ranged from $27.5 \%$ to $42.7 \%$ with a mean of $35.8 \%( \pm 3.8 \%)$. Pre-operative platelets ranged from 138 to $329 \mathrm{~K} / \mu \mathrm{L}$ with a mean of $239( \pm 60 \mathrm{~K} / \mu \mathrm{L})$. Table 2 details the operative and post-operative findings. The average surgeon-estimated blood loss for the group was $775 \mathrm{cc}( \pm 148 \mathrm{cc})$.

The mean time of collection was 47 seconds $( \pm 10.2)$ and the mean blood collected during this time was $110.8 \mathrm{~mL}( \pm 53.4 \mathrm{~mL})$. The mean post-operative reduction in hematocrit (pre-delivery hematocrit - postpartum day 1 hematocrit) was $4.4 \%$, and there were no blood transfusions. The blood loss per patient was standardized to one minute (blood volume $\times$ [60/collection time]). The median blood loss per minute was $150 \mathrm{~mL}$ (inter quartile range $88.8 \mathrm{~mL}, 95 \% \mathrm{CI} 109.2$ $190.4 \mathrm{~mL}$ ) and not normally distributed (Figure 1). One case involved a hysterotomy extension and this case had the most rapid rate of bleeding $(186 \mathrm{~mL}$ 
Table 1. Patient demographics.

\begin{tabular}{lc}
\hline \multicolumn{1}{c}{ Characteristic } & $\mathrm{n}(\%)$ \\
\hline Age (years) & $32 \pm 4.4$ \\
Nulliparous & $6(30 \%)$ \\
Multiparous & $14(70 \%)$ \\
Gestational age (mean $\pm \mathrm{SD})$ & $38.7 \pm 1.3$ \\
Pre-operative Hct (mean $\pm \mathrm{SD})$ & $35.8 \% \pm 3.8 \%$ \\
Pre-operative Plt (mean $\pm \mathrm{SD})$ & $239 \pm 60 \mathrm{~K} / \mu \mathrm{L}$ \\
Indication for cesarean delivery & \\
$\quad$ Elective Primary & $1(5 \%)$ \\
$\quad$ Scheduled Repeat & $9(45 \%)$ \\
$\quad$ Malpresentation & $3(15 \%)$ \\
$\quad$ Prior Uterine Surgery & $1(5 \%)$ \\
$\quad$ Non-reassuring fetal status & $1(5 \%)$ \\
Failure to progress & $3(15 \%)$ \\
$\quad$ Failed TOLAC & $2(10 \%)$ \\
Primary Cesarean & $7(35 \%)$ \\
Repeat Cesarean & $13(65 \%)$ \\
Labored Cesarean & $8(40 \%)$ \\
\hline
\end{tabular}

Table 2. Features of the cesarean section cases.

\begin{tabular}{cc}
\hline Characteristic & Average \pm standard deviation \\
\hline Post-operative Hct (mean $\pm \mathrm{SD})$ & $30.3 \% \pm 3.8 \%$ \\
Hematocrit \% change & $4.4 \% \pm 3.1 \%$ \\
Volume of collected blood & $110.8 \pm 53.4 \mathrm{~mL}$ \\
Suction time & $46.6 \pm 3.8 \mathrm{sec}$ \\
Median blood loss per minute & $150.0 \pm 86.7 \mathrm{~mL} / \mathrm{min}$ \\
Case estimated blood loss & $775 \pm 148 \mathrm{~mL}$ \\
Hysterotomy extension & 1 \\
Blood transfusions & 0 \\
\hline
\end{tabular}

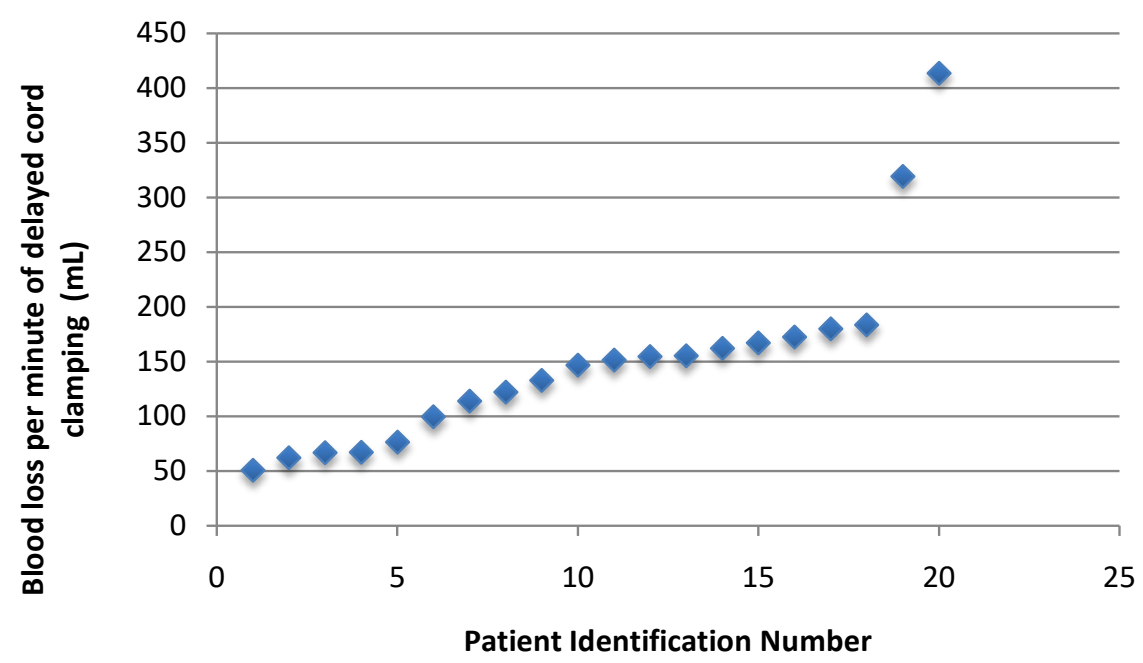

Figure 1. Hysterotomy blood loss per minute from the hysterotomy in the case series. 
in 27 seconds) and highest quantitated rate of blood loss at $413 \mathrm{~mL}$ per minute of blood.

\section{Discussion}

This prospective pilot study aimed to quantitatively assess blood loss from the hysterotomy between creation and closure during cesarean delivery at a single academic tertiary care center. In the absence of a hysterotomy extension, the quantified blood loss from the hysterotomy between creation and closure was $150 \mathrm{~mL} / \mathrm{min}$. However, in one case of a hysterotomy extension, maternal blood loss per minute increased nearly three-fold. This is the first report to quantify the blood loss between creation and closure of the hysterotomy during a cesarean delivery.

There is limited data that quantifies blood loss during cesarean delivery as a function of time. Noah et al. compared 34 patients undergoing an ex utero intrapartum treatment (EXIT) procedure in which a stapler was used to seal the hysterotomy edges to 52 non-laboring patients undergoing non-emergent cesarean delivery and found that there was no difference in post-delivery hemoglobin change [6]. A recent clinical trial by Purisch et al. randomized 113 patients to immediate cord clamping or delayed cord clamping. Change in hemoglobin in the delayed cord clamping group was $1.9 \mathrm{~g} / \mathrm{dL}$ compared to a change in hemoglobin of $1.8 \mathrm{~g} / \mathrm{dL}$ in the immediate cord clamping group, a result not statistically different when powered to detect a $0.9 \mathrm{~g} / \mathrm{dL}$ difference maternal hemoglobin [1]. This study was limited in that it included only patients undergoing scheduled cesarean deliveries, and cannot be extrapolated to labored cesareans, which are more likely to be complicated by hysterotomy extensions. In prior studies, hysterotomy extensions are associated with increased blood loss, longer operating time, and evaluations for lower urinary tract injury [7].

A strength of this study was our method of blood collection from the hysterotomy site. Although some degree of contamination from amniotic fluid may be unavoidable, we minimized this by excluding sponges that were used prior to the hysterotomy creation and including only dry sponges placed on the site after the time of delivery and during umbilical cord clamping. This meticulous methodology allowed us to isolate uterine-based bleeding during a well-defined time period. Limitations of this study include the small sample size, lack of standardization of cesarean delivery practices among surgeons, and that blood lost at the time of hysterotomy creation and active closure was not captured by our methods. Additionally, oxytocin infusion is initiated at time of cord clamping in a standardized way at our institution, and there were no cases of postpartum hemorrhage from uterine atony in the case series. Finally, we noted an increased rate of blood loss with a concurrent hysterotomy extension, but no conclusions can be drawn from a single case.

\section{Conclusion}

In conclusion, this pilot study provides preliminary data about the rate of blood 
loss from the hysterotomy during cesarean delivery. Based on our findings, the median blood loss from an uncomplicated hysterotomy was approximately 150 $\mathrm{mL}$ of blood per minute, and our data suggest that this may be much higher in the setting of a hysterotomy extension. Our findings suggest that attention to and control of hysterotomy bleeding from large sinuses or possible extensions is important and should factor into the decision to delay umbilical cord clamping or cord blood collection for banking. If there is pre-existing maternal anemia or rapid bleeding, consideration for immediate cord clamping or deferment of cord blood banking is warranted. This study provides preliminary data about the blood loss associated with this component of a cesarean delivery and will help surgeons in considering hysterotomy management and related blood loss implications.

\section{Declarations}

- The authors have no financial holdings or financial compensation for this study; however Michaela Farber received support from Gauss Surgical in the form of provision of electronic scales to measure estimated blood loss, which were used in this study. Gauss Surgical had no role in the study design, collection of data, analysis, interpretation of the data, writing of the report, nor influence on the decision for submission. The other authors have no potential conflicts of interest.

- There was no funding received for this project.

- Portions of the study were presented at The Pregnancy Meeting on February $14^{\text {th }}, 2019$ in poster format in Las Vegas, NV.

\section{Author Contributions}

All authors contributed to the design, collection, preparation, revision, and content of the present article.

\section{Conflicts of Interest}

The authors declare no conflicts of interest regarding the publication of this paper.

\section{References}

[1] Purisch, S.E., Ananth, C.V., Arditi, B., Mauney, L., Ajemian, B., Heiderich, A., Leone, T. and Gyamfi-Bannerman, C. (2019) Effect of Delayed vs Immediate Umbilical Cord Clamping on Maternal Blood Loss in Term Cesarean Delivery: A Randomized Clinical Trial. JAMA, 322, 1869-1876. https://doi.org/10.1001/jama.2019.15995

[2] Villeneuve, M.G., Khalifé, S., Marcoux, S. and Blanchet, P. (1990) Surgical Staples in Cesarean Section: A Randomized Controlled Trial. American Journal of Obstetrics and Gynecology, 163, 1641-1646. https://doi.org/10.1016/0002-9378(90)90643-L

[3] Gilson, G.J., Kephart, W.H., Izquierdo, L.A., Joffe, G.M., Qualls, C.R. and Curet, L.B. (1996) Comparison of Absorbable Uterine Staples and Traditional Hysterotomy 
during Cesarean Delivery. Obstetrics \& Gynecology, 87, 384-388. https://doi.org/10.1016/0029-7844(95)00438-6

[4] Burkett, G., Jensen, L.P., Lai, A., O'Sullivan, M.J., Yasin, S., Beydoun, S. and McLeod, A.G. (1989) Evaluation of Surgical Staples in Cesarean Section. American Journal of Obstetrics and Gynecology, 161, 540-545; Discussion 545-547. https://doi.org/10.1016/0002-9378(89)90353-0

[5] Hoskins, I.A., Ordorica, S.A., Frieden, F.J. and Young, B.K. (1991) Performance of Cesarean Section Using Absorbable Staples. Surgery, Gynecology \& Obstetrics, 172, 108-112. https://doi.org/10.1097/00006254-199109000-00010

[6] Noah, M.M.S., Norton, M.E., Sandberg, P., Esakoff, T., Farrell, J. and Albanese, C.T. (2002) Short-Term Maternal Outcomes That Are Associated with the EXIT Procedure, as Compared with Cesarean Delivery. American Journal of Obstetrics and Gynecology, 186, 773-777. https://doi.org/10.1067/mob.2002.112249

[7] Giugale, L.E., Sakamoto, S., Yabes, J., Dunn, S.L. and Krans, E.E. (2018) Unintended Hysterotomy Extension during Caesarean Delivery: Risk Factors and Maternal Morbidity. Journal of Obstetrics and Gynaecology (Lahore), 38, 1048-1053. https://doi.org/10.1080/01443615.2018.1446421 